Erik Esche, David Müller, Robert Kraus, Günter Wozny

\title{
Systematic approaches for model derivation for optimization purposes
}

Journal article | Accepted manuscript (Postprint)

This version is available at https://doi.org/10.14279/depositonce-11370

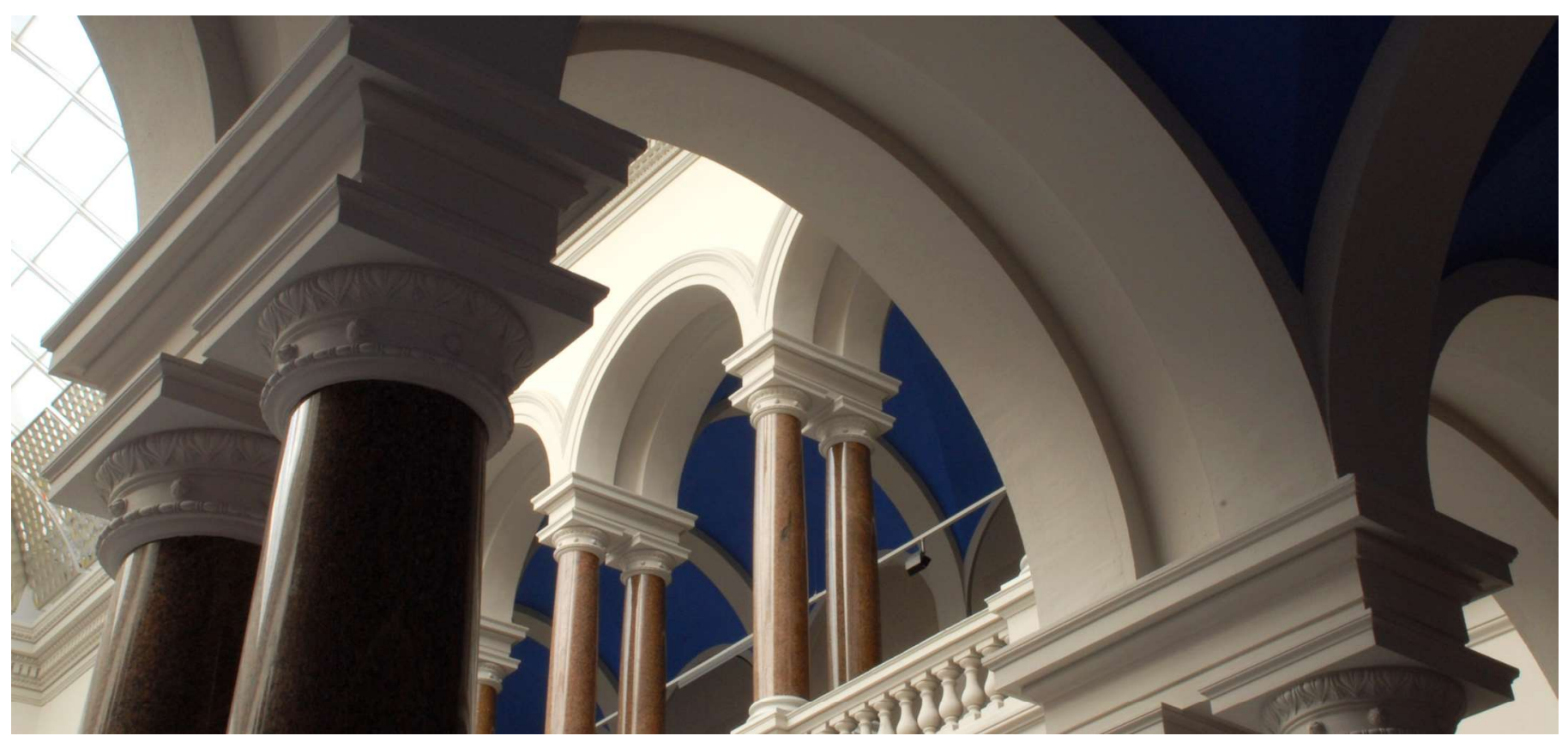

Esche, E., Müller, D., Kraus, R., \& Wozny, G. (2014). Systematic approaches for model derivation for optimization purposes. Chemical Engineering Science, 115, 215-224.

https://doi.org/10.1016/j.ces.2013.11.041 


\title{
Systematic Approaches for Model Derivation for Optimization Purposes
}

\author{
Erik Esche and David Müller, Robert Kraus, Günter Wozny \\ Berlin University of Technology, School of Process Sciences, Chair of Process Dynamics and \\ Operation, Sekr. KWT-9, Straße des 17. Juni 135, D-10623 Berlin, Germany
}

\begin{abstract}
One of the main problems in process optimization lies in the non-linearity, nonconvexity, and sheer size of existing process models. In this contribution, a systematic workflow for process systems engineers developing models suitable for optimization purposes is presented. Hereby, three fundamentally different cases are discussed: the availability of a complex, highly accurate model; the existence of a simplifying, so called short-cut model; and the non-existence of a model of any kind. Furthermore, a focus is lain on the systematic model reduction for complex systems by means of linearization and convexification. Afterwards, two case studies are presented showing how this workflow can be applied to a reactive absorption system and to a multi-phase separation process. The presented systematic leads to a successful implementation of process models applicable for optimization, which are both reduced in size, non-linearity, and non-convexity.

Keywords: Model Derivation, Process Optimization, Convexification, Linearization, Short-cut Model
\end{abstract}

\section{Introduction}

"The solution of very large-scale nonlinear programming problems still remains pretty much an art.” - Stratos Pistikopoulos, International Conference on Process Systems Engineering Asia - June 2013

Email address: erik.esche@tu-berlin.de, david.mueller@tu-berlin.de (Erik Esche and David Müller, Robert Kraus, Günter Wozny)

URL: www.dbta.tu-berlin.de (Erik Esche and David Müller, Robert Kraus, Günter Wozny)

submitted to Chemical Engineering Science 
During the last few decades great advancements in solving large-scale non-linear and other programming problems have been made. Despite the vast evolution of the algorithms, a large portion of the actual success to solve a programming problem still depends upon the skill of the programmer or process systems engineer preparing the model for optimization. In this contribution, the authors take a step to systematize the model preparation and thus demystify this "art".

\subsection{Motivation}

Applied online-optimization of industrial plants has become an increasingly prominent topic. Several examples of successful implementation of model-based optimization from the petro-industry such as (Sarabia et al., 2012) for hydrogen networks, (Jalilova et al., 2008) and (Ramdial et al., 2009) for refineries, and (Müller et al., 2012b) for the chemical industry are presently available. The success of these model-based optimizers is due to the utilized models with two important characteristics: very high to reasonable accuracy regarding the process and acceptable computational effort regarding the optimization calculation. In chemical engineering a multitude of applications ranging from distillation to reaction to absorption exist. In many cases, a model of the system is desired, either for simulation, optimization, or advanced control purposes. Often, engineers will find themselves in one of the following three situations:

- Deterministic models of systems are available, which describe the phenomena, process units, or entire plants with high accuracy. Due to their nonconvexity, non-linearity, and sheer size in terms of equations and variables, application of these models for optimization purposes is seldom seen.

- In contrast to these highly accurate models stands the availability of simplified standard models, such as a continuously stirred tank reactor models consisting of integral mass and energy balances. What these models make up for in computational time, they lack in accuracy. Their viability for optimization can usually be guaranteed, but the results are scarcely ever sensible.

- Finally, the possibility of a case with no model, no detailed kinetics, no or inconsistent thermodynamic data, or simply a not so well known system has to be taken into consideration.

Each situation requires its own particular strategy to formulate or rework the model. 


\subsection{Goal}

The goal of this contribution is to present a systematic workflow for process systems engineers developing models suitable for optimization purposes. Hence, strategies are presented to reduce the size, non-convexities, and non-linearities in exact, but too expensive models, to adjust rough models to real behavior regarding phenomena or whole processes of actual plants, and to develop completely new models for unknown, novel systems. Different paths and decisions are taken into account, depending on the information available for the model development. Furthermore, two examples are given on how this methodology can be applied.

\section{Status Quo}

Most optimization in process systems engineering is carried out with gradientbased algorithms. ${ }^{1}$ First or second order Taylor polynomials on a Lagrange reformulation of the optimization problem are used. The algorithms converge at the fulfilled KKT conditions (Kuhn \& Tucker, 1951). Due to the Lagrange reformulation, the optimization algorithm is handed the entire scope of the model, at least in case of simultaneous optimization. For all derivative free, stochastic, or swarm theory based algorithms this is not true. In this contribution, the focus will lie on the former, meaning that continuously differentiable models are to be preferred at all times.

At the moment optimizers follow several different strategies to set-up and solve optimization problems. Among these are the application of short-cut models, model derivations based on neural networks and support vector regression, as well as the development of reduced-order models with help of principal component analysis or principal orthogonal decomposition. The pros and cons of these strategies will be discussed briefly in the following.

\subsection{Classical Short-Cut Models}

Short-cut models for process development in chemical engineering date back as far as the early 1920s. Maybe the most well-known among them is the McCabeThiele method for graphically designing distillation columns (Mccabe \& Thiele, 1925), the basis of which were presented by Ponchon, Rodebush, and Savarit

\footnotetext{
${ }^{1}$ This can be seen from the statistics of the NEOS server http://www.neos-server.org/ neos/, which clearly show a predominance of gradient-based algorithms. Statistics were analyzed on the $1^{\text {st }}$ of August 2013.
} 
(Gomes, 2007) in 1921, 22, and 23 respectively. Whilst the authors of these shortcut models claim a fast and accurate calculation, the actual offset to the real application is usually quite big and the applicability rather small. A simple example is, that McCabe-Thiele's method is only valid for components with equal molar heat of vaporization. Given their nature, the computational complexity of shortcut models is quite low and convergence can usually be guaranteed. Transferring a short-cut model for one application to a second often poses a challenge.

However, short-cut models are often a good starting point for a basic model derivation for optimization purposes. By adapting to real plant data their low accuracy might be rectified for a certain region of interest.

\subsection{Neural Networks and Support Vector Regression}

Nandi et al. (2004) focus on the development of empirical models for process optimization looking at both artificial neural networks (ANN) and support vector regression (SVR). ANNs can be constructed based solely on process data, no phenomenologic knowledge is required, and multivariate dependencies can easily be described. The training of the ANN usually involves a highly non-linear and non-convex objective function (e.g. minimization of the sum of least squares). Therefore, a lot of effort has to be put into finding the global optimum, guaranteeing a close fit between data and model. SVRs are similarly exclusively based on process data. The objective function is quadratic and allows for the direct calculation of the global minimum. Both ANN and SVR models can directly be used for optimization purposes, without reformulations required. However, during the synthesis process of each model type neither smoothness, nor continuity, nor differentiability can be enforced. Consequently, ANN's and SVR's can be highly impractical for the application of gradient-based optimization algorithms. Additionally, it has to be remarked, that for both model types the extrapolation to regions, for which no process data was obtained, is inadvisable. Their derivation strategy allows no insight into how the models are going to perform in those regions they were not trained in (Bishop, 1994).

\subsection{Reduced Order Models}

Up to now, optimization on expensive computational fluid dynamics (CFD) models is scarcely ever performed. The main reason is the time required for each simulation step. Lang et al. (2009) present an approach for applying principal component analysis (PCA) to derive reduced order models (ROMs) for process optimization. The great advantage of the derived model lies in the computational time it takes to solve it, i.e. in general a few seconds instead of a couple of hours 


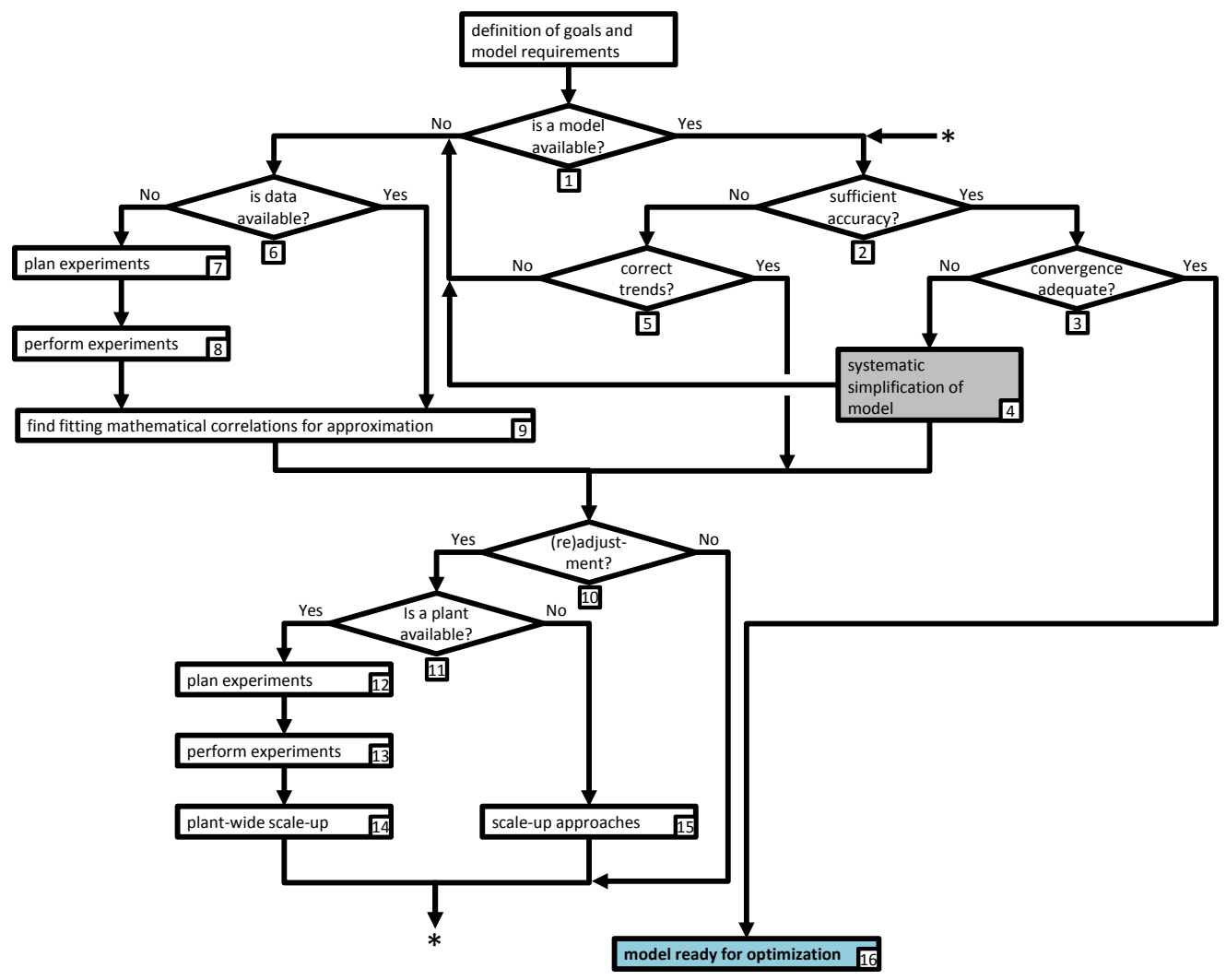

Figure 1: Systematic workflow for the development of models for optimization.

or even days for a CFD model. Given the structure of the PCA, or principal orthogonal decomposition (POD) for dynamic systems, the points at which the ROM is trained is accurately described. However, closer investigations by Lang et al. (2009) showed that "there is considerable scope for future work in ROM development" seeing as it is of the utmost importance how densely and widely the ROM is fitted to the full-scale model, in this case the CFD. The hence developed model can have the same issues as ANNs and SVRs regarding their accuracy in interpolation and extrapolation.

\section{Optimization Model Development}

\subsection{Workflow}

The workflow to design a model suitable for optimization purposes is presented in Figure 1. Since several of the steps can be taken multiple times or left 
out for certain cases, each step is discussed individually as opposed to discussing one linear path. Two certain paths are later shown in the case studies.

Before starting down the workflow, a definition of goals and model requirements is needed. As an example: A goal can be the exact description of a dynamic system for the application of a real-time optimizer for advanced control strategies. This means, an accurate, but computationally inexpensive, fast converging model is desired. Hence, the model requirements are set: non-linearities as well as nonconvexities must be avoided wherever possible to prevent the frequent appearance of local infeasibilities.

With these requirements in mind, a model has to be found. This leads to the first step: a query for the existence of a model. If a model for the phenomena, process unit, unit cluster, or entire process exists, then the next step is number 2. Often, models can easily be generated by systematic investigation of the system of interest. Several theoretical systematics, e.g. as discussed in (Marquardt, 1996), (Rodrigues \& Minceva, 2005), or (Heitzig et al., 2011) as well as programs such as ModDev presented in (Jensen \& Gani, 1999), exist. These aid the process systems engineer in deriving the process models. Otherwise, if the phenomena has not yet been described and the creation of a rigorous model is not possible, a model needs to be generated empirically (step 6).

In step 2, the accuracy of the model has to be checked. In general, this means that the outputs relevant for the optimization formulation need to be described as accurately as possible. Such a validation can be performed based on measurement data of the real application. If the accuracy of the model is sufficient, then the convergence behavior has to be analyzed in step 3. Otherwise, the model has to be further analyzed in step 5. Of assistance here can be a global sensitivity analysis Homma \& Saltelli (1996). The sensitivity of the model output with respect to the values of the input variables can thus be checked.

In step 3, the convergence behavior of the model is analyzed. Possible methods for this analysis include the test of various initial conditions or data sets for the optimization problem and the comparison of the respective number of iterations, CPU times in NLP-evaluations and appearance of local infeasibilities. If the behavior is satisfactory, then the model is ready for optimization (step 16). Otherwise, a systematic simplification of the model is required (step 4). This step is more extensive and will be discussed later on.

Step 5 analyses the existing model with respect to its display of trends shown by the real application. In many cases, the absolute values of outputs are only of limited relevance. Especially for optimization purposes the gradients, hence the position of minima depending on the controls are more important. If the trends 
are displayed correctly, a further readjustment to the real application can lead to a correct representation of the desired application. If the trends are off-target, then step 6 should be faced.

In step 6, the existing, research project-specific data pool of experimental knowledge has to be evaluated. Yet again, the extend of information on the outputs with respect to all control variables is of importance. To allow for the derivation of an exact empirical model based on experimental data, the values of all outputs are required for a wide sampling over the input space. Sometimes, it is more sensible to also chose additional intermediate outputs to limit the experimental effort. For example, if the information for fluid properties, such as viscosities, heat capacities, etc., are already available, experiments to find those can be avoided by incorporating those experimental data or existing correlations. Depending on how extensive the existing sampling over the input space is, either steps 7 (further experiments required) or 9 have to be pursued.

The planning and execution of experiments is done in step 7 and 8. In this contribution, we refrain on giving details on experimental design and point the reader to extensive literature such as (Anderson \& Whitcomb, 2000) or (Montgomery, 2013). In general terms, however, the structure should be as follows: First of all, a definition of the desired correlations or dependencies is required. At this point is has to be decided, which state and control variables are of importance for the description of the system. This includes inputs as well as outputs. Lastly, the range of and the density of the sampling over the space of influencing variables has to be set. Obviously, respective steps known in design of experiments or optimal experimental design can be taken.

No matter where the experimental data stems from, the development of the describing correlations should preferably be done as follows: Seeing as humans prefer to deal with two- or three-dimensional correlations, bivariate dependencies of outputs on their inputs should be analyzed initially. Based on these dependencies, fitting mathematical functions can be found. Further influences of additional inputs can then be included as functions for the parameters in bivariate correlations. If this approach fails for higher dimensions of the input space, alternative strategies such as multivariate linear regression can be chosen. The correlations should then be implemented into a first principle model in the form of mass balances.

Step 10 discusses the necessity for a refitting of an existing model to a larger system, e.g. lab- to pilot plant-scale. In case the initial experiments or the initial model derivation were already carried out based on the full-scale application, the fitting can be skipped and step 2 needs to be revisited. Otherwise, step 11 is taken. 
Query 11 is highly dependent on the available experimental facilities. If a real-sized plant is available, experiments should be planned to further adjust the existing model to that system (see steps 12 to 14). If not, step 15 advises on how to proceed.

Steps 12 and 13 are the equivalent to steps 8 and 7 with the basic difference in the application size.

Based on the experiments in 12 and 13, step 14 foresees the adjustment of the model to the larger scale. The larger size usually implies that equilibria are not attained or heat loss occurs, and hence adjusting factors have to be introduced. These are either constant or can be described using basic correlations. In any case, the adjustments of the model have to be tested with respect to their accuracy and convergence behavior returning to step 2 .

In case no experimental facility is available for the larger scale, standard heuristics for scale-up and numbering up can be applied instead. Since the applications for scale-up techniques are so numerous, that further considerations at this point are omitted.

Once the derived or existing model fulfills both the requirements for accuracy and convergence behavior, it is ready to be used for optimization purposes.

\subsection{Systematic Model Simplification}

To effectively perform a simplification of an existing model, the following structural dissection is proposed. First of all, the model is separated into six distinct parts. These are mass and component balances (M), equilibria formulations (E), summations (S), energy balances $(\mathrm{H})$, momentum balances (I), and auxiliary equations (Aux). In the following, these will be defined and discussed in detail. For each part convergence problems and possible strategies to alleviate them are presented.

Mass and Component Balances: In standard models, there are several different types of mass and component balances, each with their distinct issues to the complexity and convergence behavior of the model. Differentials in balances are handled separately.

- The total mass balance is usually linear and therefore requires no simplification.

- The total mole balance, on the other hand, may contain source terms through e.g. reactions. The non-linearities normally lie within the reaction kinetics, which will be dealt with in the auxiliary equations. 
- Component balances in terms of moles or mass may also include source terms. Apart from dealing with the non-linearity of the kinetics separately, they may completely be avoided by reformulation the component balances as atom balances. In case of trace components, a differentiation between vital and superfluous components should be done. The latter can simply be left out, the former could be required for kinetic calculations and should therefore be scaled manually to avoid singularities in the Jacobian. By simply dividing these essential trace components by their initial or feed amounts, the bad scaling is alleviated. Lastly, if mole or mass fractions are employed for the formulation, the balances may be bilinear. This can be compensated by using component mole flows etc. instead.

- Atom balances may also contain bilinear formulations. These can be dealt with as above.

- The scaling of differentials, especially of order higher than one, is commonly quite challenging. As of yet no standard procedure for scaling second order differential equations is known. One way forward is the separation of a differential equation into its basic phenomena, such as convective, diffusive, dispersive, and reactive parts. In most cases, these phenomena are simply to one another and can therefore be looked at separately. By comparing the magnitude of each, their influence on the differential equation can be evaluated and those with low impact are simply left out. Of course, these simplifications have to be done carefully, always keeping the loss of accuracy in mind. This is especially important regarding the coupling between differential equations and their boundary conditions, which might also contain differential terms. On top of that, the differentials themselves need to be dealt with. Solving non-ordinary differential equations systems is usually quite challenging. Furthermore, choosing an appropriate discretization method depending on stiffness and size of the problem is also of relevance. For optimization purposes collocation methods should be preferred given their high accuracy and stability, as shown in (Arora et al., 2006) and (Biegler, 2010).

Equilibria: Equilibria are a key part of most phenomena-based models in chemical engineering. There are three fundamentally different types, which will be discussed in turn:

- Chemical Equilibria - Phase Equilibria: Phase equilibria describing vaporliquid, solid-liquid, or gas-liquid combinations are in general non-linear. 
The strongest non-linearity therein stems from the correction terms for the real fluid behavior. If the correction terms are close to one, they might as well be fixed. Otherwise one might try fixing them to their current value. It is important not to lose the real fluid behavior. If they cannot be fixed, simplifications of the auxiliary equations should be considered.

- Switch Between Phase States: A critical point in modeling phase equilibria are possible switches between types of equilibria. An example would be the switch between a vapor-liquid equilibrium (VLE) and a vapor-liquid-liquid equilibrium (VLLE). The main issue with this switch is of course, that it implies a potential non-differentiability in the model. A possible relaxation for these types of switches is the introduction of a sigmoid function which deactivates non-appropriate model parts. For the VLE-VLLE example, the condition would be the position within or without the two-phase region. To avoid the appearance of singularities in the Jacobian through these deactivated model parts, slack variables should be introduced, which ensure that at least a single variable should be active.

- Chemical Equilibria - Reaction Equilibria: Almost without exception, reaction equilibria are highly non-linear and mostly also non-convex. A first step to tackle computational issues here could be the introduction of pseudocomponents to simplify and replace kinetics. Another challenge in reaction equilibria is the appearance of singularities caused by trace components. Manual scaling, e.g. dimensionless form, change of units, scaling by magnitude, of the respective equations could ease this issue. Trace components with a low impact on the kinetics can of course also be fixed to their mean concentrations and their equations can be left out.

- Thermal Equilibria: Thermal equilibria are always linear and direct calculation should be possible.

- Mechanical Equilibria: Mechanical equilibria, implying the identity of pressures is also usually linear and can also be directly calculated.

\section{Summations:}

- Summation of Fractions: By the summation of fractions, one usually understands the sum over all mass, volume, or mole fractions. Consequently, the sum is always one and each variable also is tightly bounded between zero and one. This may lead to a slow convergence, seeing as solvers might 
continuously violate those bounds. A simple replacement by the summation of absolute quantities might ease this issue, which will hence be further discussed. A further problem that frequently appears in dynamic simulations is the handling of empty hold-ups or non-flowing streams. This can be avoided by following measures: First of all, liquid tanks are initialized with an arbitrary gas component, e.g. nitrogen. Secondly, pipes are flushed with an inert component.

- Summation of Absolute Quantities: Examples for absolute quantities are component mole flows and component hold-ups and their analogues in terms of volume and mass. Despite the wider bounds on the absolute values, one downside exists to their summation. In case absolute quantities are poorly scaled, meaning that some of them take comparatively small or large values, singularities can appear in the Jacobian matrix. Therefore, selective manual scaling of those outliers is advised.

Energy Balances: The energy balance can cause convergence problems due to it's possibly strong influence on the entire system. At this point, the energy balances will be dealt with in four parts.

- Energy balance without source term: These balances usually describe standard heat exchange. The non-linearities which might appear through e.g. the logarithmic temperature difference, can sometimes be dealt with through linearization. Scaling can be assisted by selecting the right units for the formulation.

- Energy balance with source term: Source terms usually come with appearance of reactions, absorptions, adsorptions, or similar phase change phenomena. For reactions the source term usually looks similar to Eq. 1.

$$
\sum_{i=1}^{n} \dot{r}_{i} \cdot \Delta_{R} h_{i}
$$

The non-linearities pertained in the reaction rate of each reaction $\dot{r}_{i}$ are discussed elsewhere (see auxiliary equations). To avoid the bilinearity of the sums, the heats of the reactions $\Delta_{R} h_{i}$ should be fixed to an average value depending on the expected temperatures.

- Energy balance with enthalpy of formation: The challenge herein is the fact, that the reference point for a mixture depends on the concentrations. 
Hence, additional non-linearities appear, when this formulation is chosen. An alternative is always the use of a unifying source-term, e.g. using heat of reactions or heat of absorptions instead.

- Energy balance with differentials: The same as for the mass balances with differentials applies here.

\section{Momentum Balances:}

- Constant Pressure Problems: Pressure losses are often neglected, hereby no issues should arise.

- Pressure Loss Correlations: At this point, a focus will be put upon pipe flows. Therefore, three distinctly different regimes need to be taken into regard. The standard equation for calculating the pressure loss for a given density $\rho$, flow velocity $w$, pipe length $L$, and diameter $d$ is given in Eq. 2

$$
\Delta p=\zeta \cdot \frac{\rho}{2} \cdot w^{2} \cdot \frac{L}{d}
$$

For laminar flow, the friction factor $\zeta$ therein is calculated as:

$$
\zeta=\frac{64}{R e}
$$

Hence, the direct implementation of this correlation into Eq. 2 leads to a linear dependency of $\Delta p$ with respect to $w$. This linearity should therefore be exploited. For the intermediate regime between laminar and fully turbulent flow, the correlations become highly non-linear. A simple reformulation as foreseen for the linear flow is not possible. A local linearization should be considered instead. For fully turbulent flow, $\zeta$ is usually constant, the pressure loss equation is therefore quadratic with respect to $w$, which is a nice convex function despite its non-linearity. Switching between those regimes can of course cause non-differentiabilities. If these switches are unavoidable, sigmoid functions can be used to create a smooth correlation for all regions.

- Bernoulli Equation: The Bernoulli equation consists of three parts, which are the inner energy, the kinetic, and the hydrostatic energy. In most cases, the first term can be neglected, unless chemical reactions occur. Otherwise, 
the change in the inner energy between the two points the Bernoulli equation is applied to needs to be investigated more closely.

$$
d u=c_{v} \cdot d T=d h-p \cdot d v-v \cdot d p=c_{p} \cdot d T-p \cdot d v-v \cdot d p,
$$

wherein $c_{v}$ and $c_{p}$, the specific heat capacities at constant volume and pressure respectively, are usually dependent on both temperature and composition. The key point here, is to identify which of the differentials are the most influential in the decomposition given in Eq. 4 and to fix all the others to zero or to a constant value.

The second part of the Bernoulli equation, the kinetic part, can often enough be neglected, if the cross-sectional area of the pipe is constant, no change in the density of the fluid appears, and the flow velocity stays constant. Otherwise, this quadratic, convex term stays in the equation and should not create any bigger issues. In case only the hydrostatic part remains, the Bernoulli equation turns completely linear.

- Navier-Stokes Equation: For the solution of the Navier-Stokes equation in its unabbreviated form extensive CFD simulations are required, which usually rule out any sensible form of optimization.

$$
\rho \cdot\left(\frac{\partial \vec{w}}{\partial t}+\vec{w} \cdot \nabla \vec{w}\right)=-\nabla p+\eta \cdot \nabla^{2} \vec{w}+\rho \cdot \vec{g}
$$

Several simplifications of the Navier-Stokes equation given in Eq. 5 exist. A selection of these will be revisited here.

For immobile systems, Navier-Stokes of course simply turns into the basic description of the hydrostatic field caused by external forces, e.g. gravity. This is usually a linear equation. For a vortex-free system, the NavierStokes equation can be reduced to the Bernoulli equation as discussed above. These deliberations should suffice at this point. For further simplifications, other flow regimes, or correlations refer to literature such as (Acheson, 1990) or (Kundu et al., 2012).

Auxiliary Equations: The following part discusses all auxiliary correlations which give additional information for the previously discussed sections.

- Fluid properties: For the calculation of surface tensions, viscosities, heat capacities, as well as densities basic polynomial functions usually exist describing the pure components. The most common issues arising with these 
is their bad scaling and the non-linearity. These issues can of course be overcome by manual scaling and linearization in the region of interest.

- Equations of State: A main challenge, when using equations of state, is their non-linearity and the therein possible non-convexity in addition to the non-linearity of their solution space, i.e. for all cubic equations of state. In general, the more accurately an equation of state describes the real fluid behavior, the more complex, non-linear, and non-convex it becomes. Therefore, the first question that has to be asked is what accuracy is required to model the regarded system. More simpler solutions should be preferred, if their accuracy is sufficient. Often enough cubic equations of state are implemented, which are able to describe both liquid and vapor phase with a high accuracy. Equally often enough, only one of the two possible solutions is actually required. In those cases, simpler solutions, such as the Virial equation, which only describe the vapor or the liquid phase, should be used instead. If, however, a cubic equation of state is unavoidable, inequality constraints to avoid the third root in the two-phase region should be implemented, whereby details can be found in (Kamath et al., 2010).

- $g^{E}$ Models: Only in cases, where liquid phases appear, $g^{E}$ models are required. A possible strategy to decide how to cope with the inherent complexity of $g^{E}$ models lies in analyzing the real fluid behavior by looking at the value of the natural logarithm of the activity coefficients $\ln (\gamma)$.

For values close to 0 , the $g^{E}$ model can of course simply be left out.

For moderate values larger or smaller than 0 , which do not cause the appearance of homogeneous or heterogeneous azeotropes, a simpler $g^{E}$ model might be preferable, such as Wilson's or van Laar's.

For stronger deviations from 0 , whenever azeotropes appear, one might consider choosing a less non-linear $g^{E}$ model which is still able to show the same real fluid behavior, e.g. using NRTL instead of UNIQUAC. The former's derivatives are a lot easier to compute than the latter's.

- Reaction Kinetics: Reaction kinetics pose a number of challenges, some of which can not easily be overcome. Among those are the appearance of reaction relevant trace components in reaction rates, which naturally cause bad scaling. Similarly, non-linearities are inevitable, unless the whole reaction system can be fundamentally simplified as discussed in the reaction equilibria section above. There is however, a measure to simplify the existing 
non-linearities by strategically tackling the multivariate interdependencies in reaction rate equation systems. Smaller, non-linear terms within each reaction rate can be described by a newly introduced variable, which is described in an additional auxiliary equation. This measure is shown in Eq. 6. Of course, this increases the number of equations and variables, however, the hightened sparsity of the Jacobian matrix is of advantage.

$$
\dot{r}=\frac{\overbrace{k_{0} \cdot \exp \left(-\frac{E_{A}}{R \cdot T}\right)}^{a}}{1+\underbrace{K_{e q} \cdot \exp \left(-\frac{\Delta H_{a d}}{R \cdot T}\right) \cdot c_{l}}_{b}} \cdot \overbrace{c_{m} \cdot c_{n}}^{c}=\frac{a \cdot c}{1+b}
$$

The simplifying steps laid out above are nowhere near complete. The problems in chemical and process systems engineering are so complex, that not everything can be considered herein. These steps are meant as a first thought-provoking impulse and to initiate further discussions among chemical engineers.

\section{Case Studies}

In the following, two case studies are presented, which implement the workflow described above. Firstly, the amine-based absorption of $\mathrm{CO}_{2}$ is discussed as an example for a system that is well investigated and has been modeled rigorously. Secondly, a model for the three-phase liquid separation dynamics of an oil-water-surfactant system is empirically developed.

\subsection{Amine-based Absorption of $\mathrm{CO}_{2}$}

As part of the cluster of excellence "Unifying Concepts in Catalysis" (UniCat) the oxidative coupling of methane $(\mathrm{OCM})$ to ethylene $\left(\mathrm{C}_{2} \mathrm{H}_{4}\right)$ is investigated. Given the low yield (up to 25\%) and comparatively low selectivity (50 to $90 \%$ depending on the operation conditions) the purification of the product gas is highly energy intensive. Hence, considerable effort has to be put into the synthesis and subsequent or concurrent optimization of the whole process concept.

Among the many steps and options considered for the superstructure is an amine-based absorption-desorption process. Including the absorption process into any superstructure optimization is of course a challenge. A very extensively investigated process is the absorption of carbon dioxide $\left(\mathrm{CO}_{2}\right)$ using monoethanolamine 
(MEA) solutions. The solubility of $\mathrm{CO}_{2}$ in aqueous solutions of MEA has been measured for a wide range of operating conditions as published by e.g. Shen \& $\mathrm{Li}$ (1992) and Jou et al. (1995). The development of the respective kinetics began as early as the 1960s by Clarke (1964) and Hikita et al. (1977). The currently most extensive set of reaction rate equations for the reactive absorption has been published by Aboudheira et al. (2003). Simulation studies on the absorption of $\mathrm{CO}_{2}$ have been carried out using varying degrees of complexity. Examples are the comparison with ammonia by Yeh \& Bai (1999) and Freguia \& Rochelle (2003), who modeled the absorption rigorously and fitted their model to lab-scale and field data.

Figure 2 shows a rough version of the flowsheet of the absorption desorption process considered for the OCM process concept. The flowsheet also represents an existing mini-plant at TU Berlin, which will be used for the $\mathrm{CO}_{2}$ removal. In

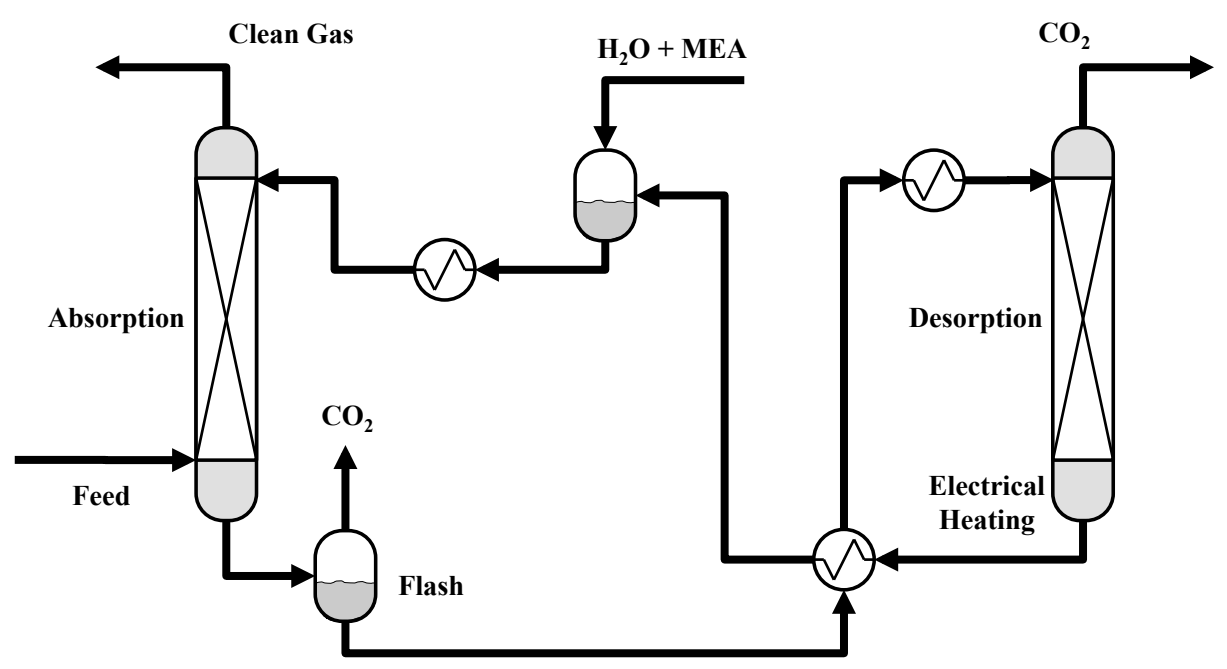

Figure 2: Rough flowsheet of the absorption desorption process for the removal of $\mathrm{CO}_{2}$ from OCM product gas.

this case study, a model is available in step 1 of the schematic in Fig. 1. In a first effort to find a fitting description for the existing mini-plant, rate-based simulations were implemented in Aspen Plus using the E-NRTL package in combination with correlations for the mass and heat transfer of the used package material. This simulation contained roughly 2000 state variables and showed a very difficult convergence behavior. First of all, closing the recycle of scrubbing liquid proved to be a challenge. It had to be done gradually, meaning slowly increasing the ac- 
tual amount of the recycled liquid and reducing the freshly supplied at the same time. Secondly, moving to a second operation point seemed to be almost impossible without having to resort to measures like reopening and reclosing the liquid cycle. Otherwise the simulations kept running into infeasibilities for various sets of initial values. On top of that, comparing the results of the Aspen simulations to experimental data from the actual mini-plant showed a sizable offset for a larger number of operation points, meaning deviations in energies required for the desorption and in the removal rate of $\mathrm{CO}_{2}$ greater than $10 \%$. Hence, it was concluded, that neither the convergence behavior nor the accuracy were satisfactory for the envisioned superstructure optimization. Hence, in step 2, the accuracy is not at a sufficient level.

Given the small diameters of both absorption and desorption columns, the offset between simulations and experiments can easily be explained by an invalidity of the applied heat and mass transfer correlations. The offset could certainly be reduced by additional model fitting. The more inconvenient problem is the source of the convergence issues. To further analyze this issue, an equilibrium-based model for MEA was implemented in AMPL and solved using both SNOPT and IPOPT. These solvers were preferred over classical NLE solvers as those scarcely ever allow for the implementation of upper and lower bounds on variables. In subsequent simulation studies, it was thus discovered, that the frequent disappearance of some ionic components such as $\mathrm{OH}^{-}$and $\mathrm{H}_{3} \mathrm{O}^{+}$, which are a vital part of the reaction kinetics, causes frequent appearances of non-differentiabilities and singularities in the kinetics, which are probably also the source of the infeasibilities already seen in the Aspen simulations. Consequently, the trends of the Aspen model (step 5) can be expected to be correct and a readjustment with an existing plant (steps 10 to 14) can be expected to be successful. Once the accuracy is achieved though (step 2), the inadequate convergence will persist (step 3). This directly leads to the simplification schematic in step 4, which will now be discussed.

Moving away from Aspen, the reduction of the size of the problem, 1200 instead of 2000 states, does not seem to solve the convergence issues. Hence, it was concluded that the source of the strong non-linearity should be tackled, which is the complexity and bad scaling of the reaction kinetics. For optimization purposes, it is not required to know about the exact make-up of all streams in a plant, especially not the concentration of every single ion. Basic equilibrium data, which shows how much $\mathrm{CO}_{2}$ is dissolved in a $30 \mathrm{wt} .-\%$ MEA solution at what temperature and pressure and what heat is generated during that process, is published by Shen \& Li (1992) and Kim \& Svendsen (2007) respectively. Their data is plotted and correlations are derived thereof. Figures 3 and 4 show the measurement and 
the fitted correlations. This relates to steps 6 to 9 in the systematic. The cor-

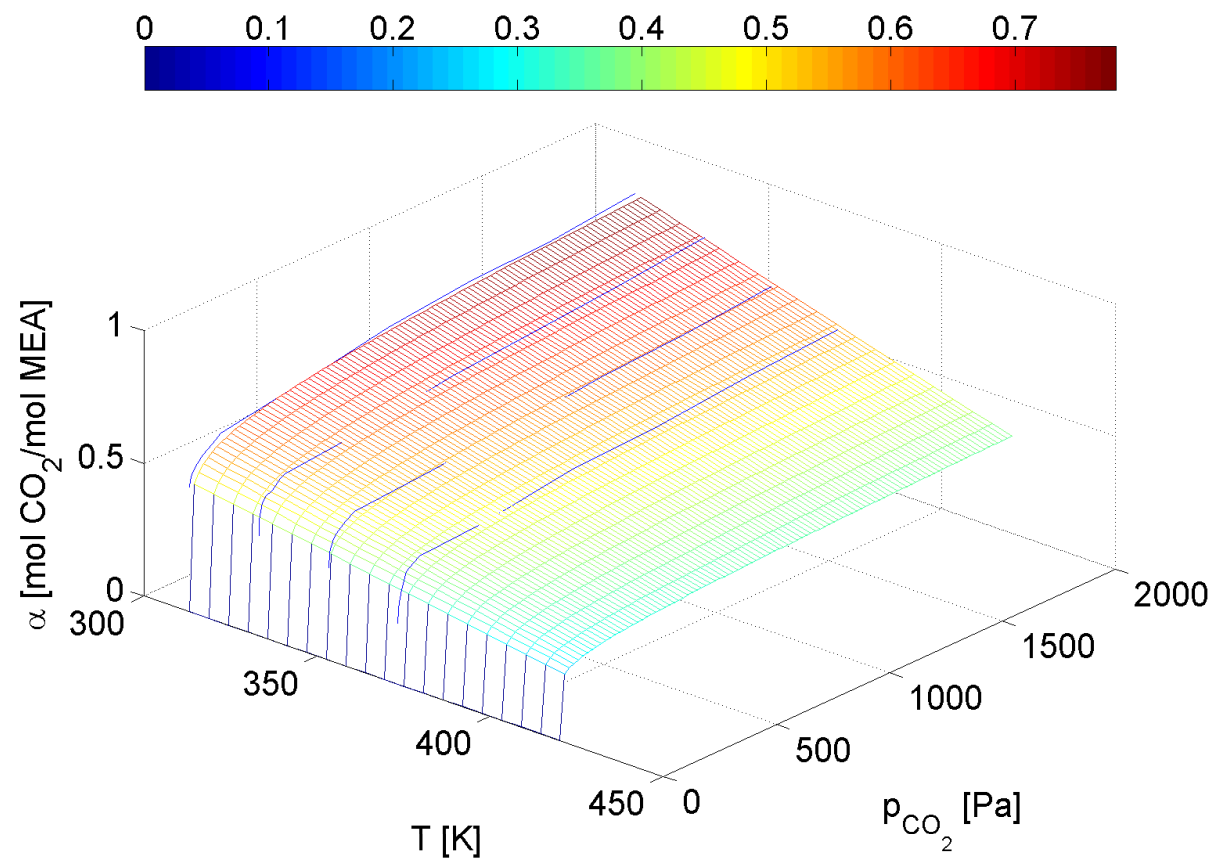

Figure 3: Solubility of $\mathrm{CO}_{2}$ in a 30 wt.- $\%$ MEA solution $\alpha$ depending on temperature $T$ and the partial pressure of $\mathrm{CO}_{2} \mathrm{p}_{\mathrm{CO}_{2}}$. The lines show the measurement data, the surface the developed correlation.

relations were derived with respect to the form and structure of the measurement data, which can easily be exploited in this three-dimensional view of the bivariate dependency. The basic form of both correlations is given in Eq. 7 and 8, wherein letters $A$ to $L$ represent the fitted parameters.

$$
\begin{gathered}
\alpha\left(T, p_{C O_{2}}\right)=(A \cdot T+B) \cdot\left(p_{C O_{2}}\right)^{C \cdot T+D}+E \\
\Delta h_{A}(T, \alpha)=(F \cdot T+G) \cdot((I \cdot T+J)-\alpha)^{K}+L
\end{gathered}
$$

Based on these correlations, a new model is developed, which uses the pseudocomponents MEA and $\mathrm{CO}_{2}$ for the liquid phase. The model consists of mole balances for water, MEA, $\mathrm{CO}_{2}$, and $\mathrm{N}_{2}$. The phase equilibrium of $\mathrm{CO}_{2}$ is described using the correlations above. Antoine's equation is implemented for the calculation of the vapor pressure of water. The energy balance has three source terms: the heat of evaporation of water, the heat of absorption of $\mathrm{CO}_{2}$, and the 


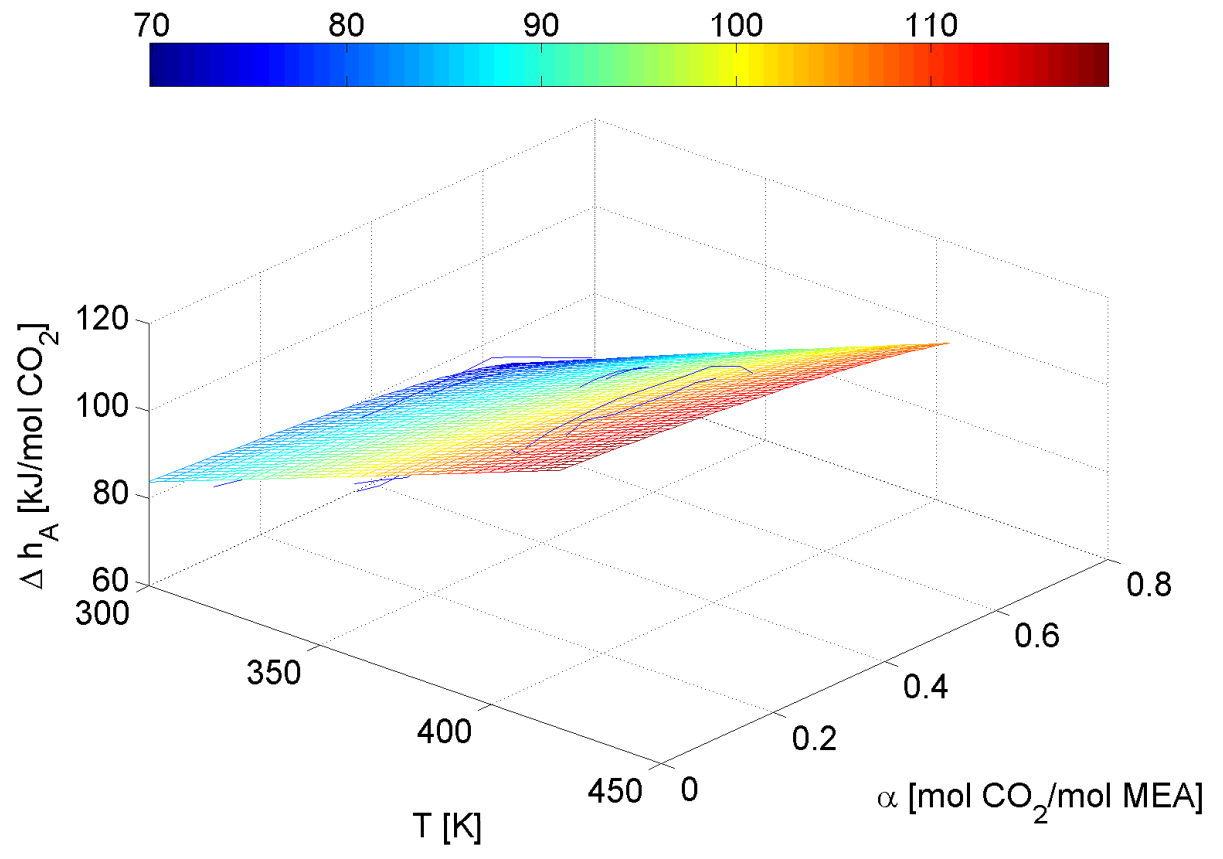

Figure 4: Heat of absorption of $\mathrm{CO}_{2}$ in a 30 wt.-\% MEA solution $\Delta h_{A}$ depending on temperature $T$ and the solubility of $\mathrm{CO}_{2} \alpha$. The lines show the measurement data, the surface the developed correlation. 
heat loss to the outside. The latter is based on a basic $U \cdot A$-correlation. Each set of equations is formulated for all equilibrium stages of the absorption column. To compensate for the constant loss of water and MEA especially in the desorption column, the liquid recycle is formulated as a simple transfer of the $\mathrm{CO}_{2}$ load after the desorption to fresh new MEA solution. The simplified new model consists of 40 stages for the desorption column and a single one for the desorption. This concludes both steps 4 and 9 and leads to additional readjustment step 10 .

This newly derived model shows an exceptionally good behavior in terms of computation time and convergence. To model the actual plant of course, further modifications are required. Seeing as a plant is available (step 11), measurement data can be generated (steps 12 to 14). For this purpose, experiments were carried out in the mini-plant varying the absorption pressure between 5 and 32 bar, the gas load factor between 0.25 and $0.42 \mathrm{~Pa}^{0.5}$, the feed concentrations of the carbon dioxide between 0.14 and $0.26 \mathrm{~mol} / \mathrm{mol}$, the $\mathrm{CO}_{2}$ removal rate between 70 and $100 \%$, as well as the flow of the scrubbing liquid from 10 to $60 \mathrm{~kg} / \mathrm{h}$. To compensate for the fluid dynamics in both columns, efficiencies were introduced into the $\mathrm{CO}_{2}$ solubility equations, the $U \cdot A$-term is fitted, and the parameters $A$ to $E$ are readjusted. It was found, that all parameters above can be set to constant values, while the efficiency for the desorption column can be described depending on five characteristic variables: the absorption pressure, the gas load factor, the feed concentration of $\mathrm{CO}_{2}$, the removal rate of $\mathrm{CO}_{2}$, and the scrubbing liquid flow.

The resulting, fitted model can represent all experimental points with a margin of error of $\pm 3 \%$ with respect to the energy required per captured kilogram of $\mathrm{CO}_{2}$. Optimization studies carried out with this model showed a fast and reliable convergence from different starting points and usually converged within a second on a 64bit AMD Athlon X2 Dual Core Processor 3800+. Hence, it is obvious that both with respect to accuracy and computational time (steps 2 and 3), this model is suitable for optimization purposes.

\subsection{Dynamic Three-Phase Model}

In this example, the development of a three phase separation model for a decanter in a hydroformylation miniplant is discussed.

Within the Collaborative Research Center SFB/TR 63 InPROMPT, a process concept for the hydroformylation of higher alkenes micro-emulsions is being investigated. The reaction known as hydroformylation is the coincident addition of hydrogen and carbon-monoxide to an alkene to produce an aldehyde (linear or branched). In most cases, the linear aldehyde is desired. The general reaction equation is displayed in Fig. 5. The production of short-chained aldehydes 


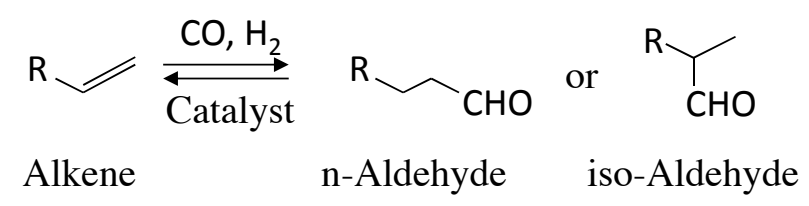

Figure 5: General hydroformylation reaction (Kupka, 2006).

from alkenes has been established as a standard industrial process. The application to alkenes longer than $\mathrm{C} 10$ in a biphasic system with a rhodium catalyst has not yet been established though. The concept currently under research at TU Berlin is focused on hydroformylation of higher alkenes in micro emulsions. A highly selective water-soluble rhodium catalyst ${ }^{2}$ is applied to react 1-dodecene to 1-tridecanal. Backbone of the process concept is the implementation of a surfactant into the process. The surfactant facilitates the mixture of the two normally immiscible liquids and thus allows a homogeneous reaction to be performed. Afterwards, the miscibility gap between the hydrophilic catalyst solution and hydrophobic alkene/aldehyde mixture is exploited. This enables the recycle of the valuable rhodium catalyst and separation of an almost pure organic product phase. The general concept for the hydroformylation in micro-emulsions is displayed in Fig. 6 (Müller et al., 2012a). To test the viability of the concept, a mini-plant has been built at TU Berlin (Müller et al., 2012a). The critical step, next to a successful reaction, is the effective phase separation. Starting at step 1 in the workflow presented in Fig. 1, the decision is made to develop a model, thus leading to step 6. Since little to no consistent thermodynamic data on the system is available, an empirical model for the phase separation must be developed and therefore continuing on to step 7.

Firstly, the required correlation is defined. Since the phase separation is to take place in a decanter, the height of each of the phases is relevant for the process. In other words, a function has to be developed that determines the height of each of the phases depending on the variables of interest. In general, these are temperature $T$, separation time $t$, and the concentrations of each of the substances present in the mixtures. The substances applied during the hydroformylation reaction are 1dodecene, the non-ionic surfactant Marlipal 24/70 (CAS: 68439-50-9), deionized water, and the product 1-tridecanal. The combination of these substances leads to three important values for describing the system:

\footnotetext{
${ }^{2}$ The catalyst applied is been sponsored by the company Unicore
} 


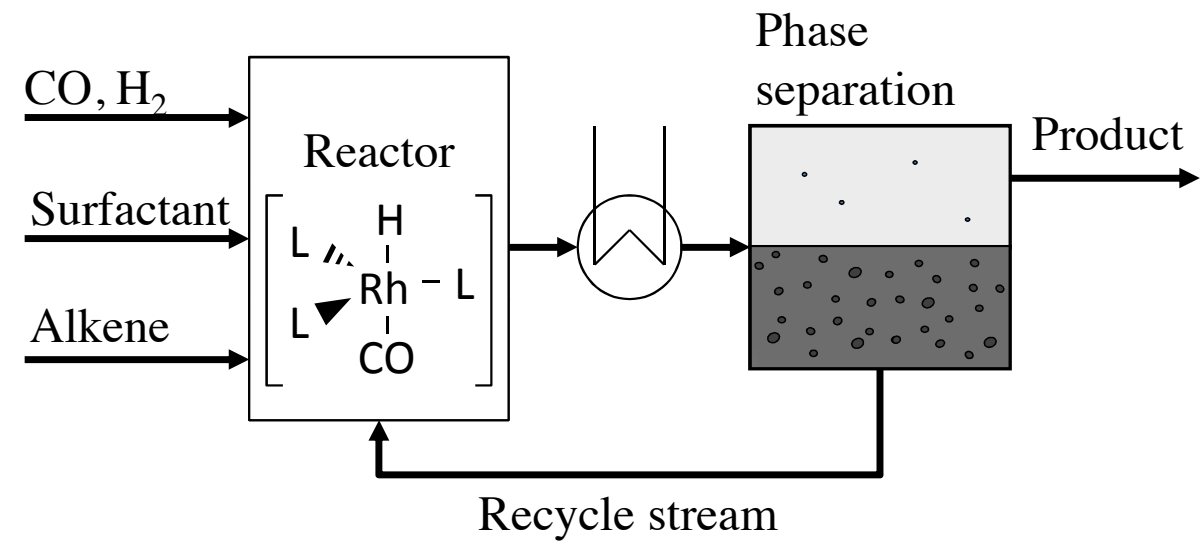

Figure 6: Process concept for the hydroformylation in micro-emulsions (Müller et al., 2012a).

- Oil-Water-Ratio:

$$
\alpha=\frac{m_{\text {oil }}}{m_{\text {oil }}+m_{\text {water }}}
$$

- Surfactant concentration:

$$
\gamma=\frac{m_{\text {surfactant }}}{m_{\text {oil }}+m_{\text {water }}+m_{\text {surfactant }}}
$$

- Product concentration:

$$
X=\frac{m_{1-\text { tridecanal }}}{m_{\text {oil }}+m_{\text {water }}+m_{\text {surfactant }}}
$$

Hereby, $m_{\text {oil }}$ is the sum of $m_{1-\text { dodecene }}$ and the product $m_{1 \text {-tridecanal }}$. Furthermore, a water soluble catalyst consisting of a rhodium-based precursor (CAS: 1487482-9) and the ligand Sulfoxantphos (sulfonated form of Xantphos, CAS: 16126503-8) are used. Thus, a total of six variables is to be implemented into the phase separation function: $h=f\left(\gamma, \alpha, X, t, T, c_{\text {catalyst }}\right)$.

Next, the range of interest for each of the variables is defined. For this, the starting point in terms of concentrations must be determined. The reaction step of the hydroformylation process is started with an $\alpha$ value of $50 \%$, a $\gamma$ value of $8 \%$, a precursor concentration of $300 \mathrm{ppm}$, ligand concentration of $4500 \mathrm{ppm}$, and a product concentration $X$ of $0 \%$ (Hamerla et al., 2013). The $\alpha$ value can vary slightly, but tends to stay in the area of $50 \%$. The decision is made to analyze 
the system within the bounds of 33 to $66 \%$. The surfactant concentration $\gamma$ is in the range of 6 to $10 \%$. Observations regarding the product concentration $X$ during batch reactions done by (Hamerla et al., 2013) show that a maximum value of $50 \%$ is adequate. Preliminary investigations have shown, that the phase separation for this system tend to be in the range of 80 to $95{ }^{\circ} \mathrm{C}$. Finally, the separation time needs to be kept at a low. The maximum time for the phase separation is set to 20 minutes.

Next up is step 8, the execution of the experiments. Hereby the region of interest of the variables is divided appropriately, in order to keep the information gain high, but amount of experiments low. For this purpose, an experimental setup discussed in (Müller et al., 2013) is designed to determine the phase heights at various temperatures and time steps. An example of the experimental results is displayed in (Müller et al., 2013).

One example for variation of parameters is displayed in Fig. 7. The surfactant concentration $\gamma$ is varied between 7, 8, and 9wt.\% for a fixed $\alpha$ value of 50\%, Product concentration $X$ of $50 \%$, a precursor concentration of 298ppm, and ligand concentration of $4500 \mathrm{ppm}$. In Fig. 7 the relative height of the product phase at various temperatures after 20 minutes of phase separation is displayed. A constant shift of $5^{\circ} \mathrm{C}$ towards higher temperatures can be observed, the lower the surfactant concentration is. With similar experiments performed for various $\alpha$ and $X$ values, correlations for the phase separation can be determined (step 9). Firstly, the results at 20 minutes are analyzed and bivariate dependencies determined. It is apparent that a combination of sigmoid functions is capable of displaying the separation zone. Thus, a function displayed in Eq. 12, is developed, whereby $p_{i}$ are to be fitted parameters.

$$
h(T)=\frac{p_{1}}{1+p_{2} \cdot e^{p_{3}-T}}+\frac{p_{4}}{1+p_{5} \cdot e^{p_{6}-T}}
$$

Secondly, the separation is regarded over time. Also here, a sigmoid function is adequate for describing the separation behavior.

$$
h(t)=\left(100-\frac{p_{7}}{1+p_{8} \cdot e^{p_{9}-t}}\right)
$$

In a third step, the influence of each of the concentrations, as presented in the example in Fig. 7, is implemented into the parameters $p_{i}$. An example is the linear temperature shift for different $\gamma$ values, which is implemented into the parameter $p_{3}$ and $p_{6}$.

$$
p_{3}(\gamma)=p_{10} \cdot \gamma+p_{11} \quad p_{6}(\gamma)=p_{12} \cdot \gamma+p_{13}
$$




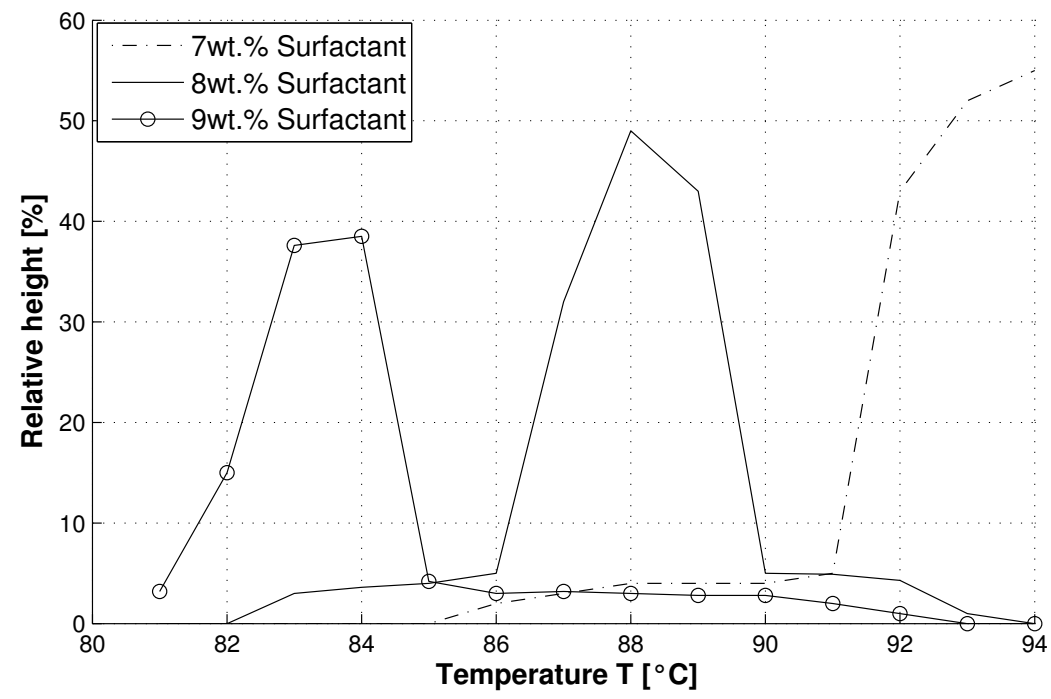

Figure 7: Relative height of the product phase for different surfactant concentrations, $\alpha=50 \%, X$ $=50 \%$, Rhodium precursor $=298 \mathrm{ppm}$, Ligand $=4500 \mathrm{ppm}$.

This systematic analyses of each of the dependencies aids in designing the separation function. The phase separation function $h(t, T, \gamma, \alpha, X)$ is then transformed to fit into a cylinder and implemented into a first principle model for the whole decanter, the results of which are displayed and applied in (Müller et al., 2013).

In general, the accuracy of the model for application in the process is high (average absolute error is $\leq 0,9 \%$ total height for each of the data points). A validation with the decanter in the miniplant has been successful. Thus, the model is ready for optimization and can easily be applied given its explicit structure.

\section{Conclusions and Outlook}

The purpose of this article is to present guidelines for process systems engineers, who are facing the task of preparing their models for optimization. Hereby, a systematic workflow is outlined to help develop, simplify, or modify models. Main focus lies on the reduction of convergence challenges such as non-linearities and non-convexities. Additionally, two case studies are discussed in which this systematic has successfully been applied and have been used for optimization.

The authors realize that the field of chemical engineering is wide and therefore many special modeling solutions exist. This article does not claim to solve all 
uprising challenges, but is supposed to be a first step in the direction of systematizing the "art" of preparing models for solving large scale non-linear programming problems. Additionally, parallel computing strategies have not been discussed in this contribution. By analyzing the structure of equation systems, e.g. by the Dulmage-Mendelsohn decomposition Dulmage \& Mendelsohn (1963), blocks of equations can be identified, which could be computed independently and in parallel to other blocks. Thus, a significant decrease of the computation time is attainable. Among others, the treatment of inequality constraints and the formulation of the objective functions need to be further discussed.

As a next step, this systematic will be implemented into the web-based modeling environment MOSAIC ${ }^{3}$. MOSAIC, more closely described in (Kuntsche et al., 2011), is a platform to facilitate the creation of, reuse of, and collaboration on models and measurement data. The environment will be extended to support measurement data evaluation and parameter identification. Furthermore, an assessment of the model validity and accuracy is to be implemented. This will be appended to each model as meta-information. Additionally, a forum will be set up on the web-page, to allow for discussions on the systematic and how to further extend it.

\footnotetext{
${ }^{3}$ www.mosaic-modeling.de
} 


\section{Nomenclature}

$\begin{array}{lll}c_{p} & \text { heat capacity at constant pressure } & \mathrm{kJ} / \mathrm{kmol} \cdot \mathrm{K} \\ c_{v} & \text { heat capacity at constant volume } & \mathrm{kJ} / \mathrm{kmol} \cdot \mathrm{K} \\ d & \text { diameter } & \mathrm{m} \\ g & \text { gravitational acceleration } & \mathrm{m} / \mathrm{s}^{2} \\ h & \text { enthalpy } & \mathrm{kJ} / \mathrm{mol} \\ h & \text { relative height } & \% \\ \Delta h_{A} & \text { heat of absorption } & \mathrm{kJ} / \mathrm{mol} \\ \Delta_{R} h_{i} & \text { heat of reaction for reaction } i & \mathrm{~kJ} / \mathrm{mol} \\ L & \text { length } & \mathrm{m} \\ m_{j} & \text { mass of component } j & \mathrm{~kg} \\ \Delta p & \text { pressure difference/loss } & \mathrm{Pa} \\ p_{k} & \text { parameter number } k & - \\ R e & \text { Reynolds number } & - \\ \dot{r}_{i} & \text { reaction rate of reaction } i & \mathrm{~mol} / \mathrm{s} \\ t & \text { time } & \mathrm{s} \\ T & \text { temperature } & \mathrm{K} \\ u & \text { inner energy } & \mathrm{kJ} / \mathrm{mol} \\ v & \text { specific/molar volume } & \mathrm{m} / \mathrm{mol} \\ w & \text { flow velocity (empty pipe) } & \mathrm{m} / \mathrm{s} \\ X & \text { conversion } & \mathrm{g} / \mathrm{g} \\ G r e e & \text { letters } & \\ \alpha & \text { CO }{ }_{2} \text { solubility } & \mathrm{mol} \mathrm{CO} / / \mathrm{mol} \mathrm{MEA} \\ \alpha & \text { water oil ratio } & \mathrm{g} / \mathrm{g} \\ \gamma & \text { activity coefficient } & - \\ \gamma & \text { surfactant mass fraction } & \mathrm{g} / \mathrm{g} \\ \zeta & \text { friction factor } & - \\ \eta & \text { dynamic viscosity } & \mathrm{Pa} \cdot \mathrm{s} \\ Q & \text { density } & \mathrm{kg} / \mathrm{m}^{3} \\ & & \end{array}$

\section{Acknowledgements}

The authors acknowledge the support from the Cluster of Excellence "Unifying Concepts in Catalysis" and the Collaborative Research Center SFB/TR 63 InPROMPT "Integrated Chemical Processes in Liquid Multiphase Systems" both coordinated by the Berlin University of Technology and funded by the German Research Foundation (Deutsche Forschungsgemeinschaft "DFG”). 


\section{References}

Aboudheira, A., Tontiwachwuthikula, P., Chakmab, A., \& Idema, R. (2003). Kinetics of the reactive absorption of carbon dioxide in high co2-loaded, concentrated aqueous monoethanolamine solutions. Chemical Engineering Science, $58,5195-5210$.

Acheson, D. J. (1990). Elementary Fluid Dynamics. Oxford University Press Inc.

Anderson, M. J., \& Whitcomb, P. J. (2000). Design of experiments. In KirkOthmer Encyclopedia of Chemical Technology. John Wiley \& Sons, Inc.

Arora, S., Dhaliwal, S., \& Kukreja, V. (2006). Application of orthogonal collocation on finite elements for solving non-linear boundary value problems. Applied Mathematics and Computation, 180, 516 - 523.

Biegler, L. (2010). Nonlinear Programming: Concepts, Algorithms, and Applications to Chemical Processes. MPS-SIAM Series on Optimization. Society for Industrial and Applied Mathematics.

Bishop, C. M. (1994). Novelty detection and neural network validation. Vision, Image and Signal Processing, IEE Proceedings -, 141, 217-222.

Clarke, J. K. A. (1964). Kinetics of absorption of carbon dioxide in monoethanolamine solutions at short contact times. Ind. Eng. Chem. Fundamen., $3,239-245$.

Dulmage, A. L., \& Mendelsohn, N. S. (1963). Two algorithms for bipartite graphs. Journal of the Society for Industrial and Applied Mathematics, 11, 183-194.

Freguia, S., \& Rochelle, G. T. (2003). Modeling of co2 capture by aqueous monoethanolamine. AIChE Journal, 49, 1676 - 1686.

Gomes, J. F. P. (2007). Reflections on the use of the mccabe and thiele method. http://www.unicap.br/revistas/revista_e/artigo6.pdf.

Hamerla, T., Rost, A., Kasaka, Y., \& Schomäcker, R. (2013). Hydroformylation of 1-dodecene with water-soluble rhodium catalysts with bidentate ligands in multiphase systems. ChemCatChem, N.A., N.A. 
Heitzig, M., Sin, G., Sales-Cruz, M., Glarborg, P., \& Gani, R. (2011). Computeraided modeling framework for efficient model development, analysis, and identification: Combustion and reactor modeling. Industrial $\mathcal{E}$ Engineering Chemistry Research, 50, 5253-5265.

Hikita, H., Asai, S., Ishikawa, H., \& Honda, M. (1977). The kinetics of reactions of carbon dioxide with monoethanolamine, diethanolamine and triethanolamine by a rapid mixing method. The Chemical Engineering Journal, 13, 7 - 12.

Homma, T., \& Saltelli, A. (1996). Importance measures in global sensitivity analysis of nonlinear models. Reliability Engineering $\mathcal{E}$ System Safety, 52, 1 - 17.

Jalilova, N., Tautiyev, A., Forcadell, J., Rodríguez, J., \& Sama, S. (2008). Production optimization in an oil producing asset - the bp azeri field optimizer case. In SPE Gulf Coast Section Digital Energy Conference and Exhibition. Society of Petroleum Engineers.

Jensen, A. K., \& Gani, R. (1999). A computer aided modeling system. Computers $\mathcal{E}$ Chemical Engineering, 23, Supplement, S673 - S678.

Jou, F.-Y., Mather, A. E., \& Otto, F. D. (1995). The solubility of co2 in a 30 mass percent monoethanolamine solution. The Canadian Journal of Chemical Engineering, 73, 140 - 147.

Kamath, R. S., Biegler, L. T., \& Grossmann, I. E. (2010). An equation-oriented approach for handling thermodynamics based on cubic equation of state in process optimization. Computers $\mathcal{E}$ Chemical Engineering, 34, 2085 - 2096. 10th International Symposium on Process Systems Engineering, Salvador, Bahia, Brasil, 16-20 August 2009.

Kim, I., \& Svendsen, H. F. (2007). Heat of absorption of carbon dioxide $\left(\mathrm{Co}_{2}\right)$ in monoethanolamine (mea) and 2-(aminoethyl)ethanolamine (aeea) solutions. Ind. Eng. Chem. Res., 46, 5803 - 5809.

Kuhn, H. W., \& Tucker, A. W. (1951). Nonlinear programming. Proceedings of 2nd Berkeley Symposium. Berkeley: University of California Press, 1, 481 492.

Kundu, P. K., Cohen, I. M., \& Dowling, D. R. (2012). Fluid Mechanics. (5th ed.). Elsevier Inc. 
Kuntsche, S., Barz, T., Kraus, R., Arellano-Garcia, H., \& Wozny, G. (2011). \{MOSAIC\} a web-based modeling environment for code generation. Computers $\mathcal{E}$ Chemical Engineering, 35, 2257 - 2273.

Kupka, J. A. (2006). Hydroformylierung von 1-Octen in Mikroemulsion. Ph.D. thesis Technischen Universität Carolo-Wilhelmina zu Braunschweig.

Lang, Y., Malcina, A., Biegler, L. T., Munteanu, S., Madsen, J. I., \& Zitney, S. E. (2009). Reduced order model based on principal component analysis for process simulation and optimization. Energy $\mathcal{E}$ Fuels, 23, 1695 - 1706.

Marquardt, W. (1996). Trends in computer-aided process modeling. Computers and Chemical Engineering, 20, 591 - 609.

Mccabe, W., \& Thiele, E. (1925). Graphical design of fractionating columns. Industrial $\mathcal{E}$ Engineering Chemistry, 17, 605 - 611.

Montgomery, D. C. (2013). Design and analysis of experiments. (8th ed.). John Wiley \& Sons, Inc.

Müller, D., Esche, E., Müller, M., \& Wozny, G. (2012a). Developement of a short-cut model for three-phase liquid seperation dynamics for a hydrofromylation mini-plant. In Proceedings of the AIChE Annual Meeting 2012. Abstract accepted for oral presentation.

Müller, D., Hoang-Minh, D., Restrepo, V. A. M., Arellano-Garcia, H., Kasaka, Y., Müller, M., Schomäker, R., \& Wozny, G. (2013). Towards a novel process concept for the hydroformylation of higher alkenes: Model development and mini-plant techniques. Chemical Engineering Science, n.a., n.a.

Müller, D., Höser, S., Kahrs, O., Arellano-Garcia, H., \& Wozny, G. (2012b). Optimization of process operation strategies by combining process models with plant operating data. Chemical Engineering Transactions, 29, $1495-1500$.

Nandi, S., Badhe, Y., Lonari, J., Sridevi, U., Rao, B., Tambe, S. S., \& Kulkarni, B. D. (2004). Hybrid process modeling and optmization strategies integrating neural networks/support vector regression and genetic algorithms: study of benzene isopropylation on hbeta catalyst. Chemical Engineering Journal, 97, $115-129$. 
Ramdial, A., Hudson, N., Pike, R., Rodriguez, J., Stratman, M., \& Sama, S. (2009). Model-based optimisation of a gas production system - the bp trinidad field optimiser. In SPE Annual Technical Conference and Exhibition. Society of Petroleum Engineers.

Rodrigues, A. E., \& Minceva, M. (2005). Modelling and simulation in chemical engineering: Tools for process innovation. Computers $\mathcal{E}$ Chemical Engineering, 29, $1167-1183$.

Sarabia, D., Prada, C. D., Gómez, E., Gutierrez, G., Cristea, S., Sola, J., \& Gonzalez, R. (2012). Data reconciliationandoptimalmanagementofhydrogennetworksina petrol refinery. Control Engineering Practice, 20, 343 - 354.

Shen, K. P., \& Li, M. H. (1992). Solubility of carbon dioxide in aqueous mixtures of monoethanolamine with methyldiethanolamine. J. Chem. Eng. Data, 37, 96 -100 .

Yeh, A. C., \& Bai, H. (1999). Comparison of ammonia and monoethanolamine solvents to reduce co2 greenhouse gas emissions. Science of the Total Environment, 228, $121-133$. 